\title{
Pattern avoidance in partial permutations (extended abstract)
}

\author{
Anders Claesson $\mathbb{1}^{\llbracket}$ and Vít Jelínek ${ }^{1 \dagger}$ and Eva Jelínkováp and Sergey \\ Kitaev $^{1 \dagger}$ \\ ${ }^{1}$ The Mathematics Institute, School of Computer Science, Reykjavik University, Menntavegur 1, IS-101 Reykjavik, \\ Iceland \\ ${ }^{2}$ Department of Applied Mathematics, Charles University in Prague, Malostranské nám. 25, 11800 Praha 1, Czech \\ Republic
}

\begin{abstract}
Motivated by the concept of partial words, we introduce an analogous concept of partial permutations. A partial permutation of length $n$ with $k$ holes is a sequence of symbols $\pi=\pi_{1} \pi_{2} \cdots \pi_{n}$ in which each of the symbols from the set $\{1,2, \ldots, n-k\}$ appears exactly once, while the remaining $k$ symbols of $\pi$ are "holes".

We introduce pattern-avoidance in partial permutations and prove that most of the previous results on Wilf equivalence of permutation patterns can be extended to partial permutations with an arbitrary number of holes. We also show that Baxter permutations of a given length $k$ correspond to a Wilf-type equivalence class with respect to partial permutations with $(k-2)$ holes. Lastly, we enumerate the partial permutations of length $n$ with $k$ holes avoiding a given pattern of length at most four, for each $n \geq k \geq 1$.

Résumé. Nous introduisons un concept de permutations partielles. Une permutation partielle de longueur n avec $k$ trous est une suite finie de symboles $\pi=\pi_{1} \pi_{2} \cdots \pi_{n}$ dans laquelle chaque nombre de l'ensemble $\{1,2, \ldots, n-k\}$ apparaît précisement une fois, tandis que les $k$ autres symboles de $\pi$ sont des "trous".

Nous introduisons l'étude des permutations partielles à motifs exclus et nous montrons que la plupart des résultats sur l'équivalence de Wilf peuvent être généralisés aux permutations partielles avec un nombre arbitraire de trous. De plus, nous montrons que les permutations de Baxter d'une longueur donnée $k$ forment une classe d'équivalence du type Wilf par rapport aux permutations partielles avec $(k-2)$ trous. Enfin, nous présentons l'énumeration des permutations partielles de longueur $n$ avec $k$ trous qui évitent un motif de longueur $\ell \leq 4$, pour chaque $n \geq k \geq 1$.
\end{abstract}

Keywords: partial permutation, pattern avoidance, Wilf-equivalence, bijection, generating function, Baxter permutation

\footnotetext{
${ }^{\dagger}$ Supported by the Icelandic Research Fund, grant no. 090038011.

$\ddagger$ Supported by project $1 \mathrm{M} 0021620838$ of the Czech Ministry of Education. The work was done while the third author was visiting ICE-TCS, Reykjavik University, Iceland.
}

1365-8050 (C) 2010 Discrete Mathematics and Theoretical Computer Science (DMTCS), Nancy, France 


\section{Introduction}

Let $A$ be a nonempty set, which we call an alphabet. A word over $A$ is a finite sequence of elements of $A$, and the length of the word is the number of elements in the sequence. Assume that $\diamond$ is a special symbol not belonging to $A$. The symbol $\diamond$ will be called $a$ hole. A partial word over $A$ is a word over the alphabet $A \cup\{\diamond\}$. In the study of partial words, the holes are usually treated as gaps that may be filled by an arbitrary letter of $A$. The length of a partial word is the number of its symbols, including the holes.

The study of partial words was initiated by Berstel and Boasson [BB99]. Partial words appear in comparing genes [Leu05]; alignment of two sequences can be viewed as a construction of two partial words that are compatible in the sense defined in [BB99]. Combinatorial aspects of partial words that have been studied include periods in partial words [BB99, SK01], avoidability/unavoidability of sets of partial words [BBSGR09, BSBK ${ }^{+}$09], squares in partial words [HHK08], and overlap-freeness [HHKS09]. For more see the book by Blanchet-Sadri [BS08].

Let $V$ be a set of symbols not containing $\diamond$. A partial permutation of $V$ is a partial word $\pi$ such that each symbol of $V$ appears in $\pi$ exactly once, and all the remaining symbols of $\pi$ are holes. Let $\mathcal{S}_{n}^{k}$ denote the set of all partial permutations of the set $[n-k]=\{1,2, \ldots, n-k\}$ that have exactly $k$ holes. For example, $\mathcal{S}_{3}^{1}$ contains the six partial permutations $12 \diamond, 1 \diamond 2,21 \diamond, 2 \diamond 1, \diamond 12$, and $\diamond 21$. Obviously, all elements of $\mathcal{S}_{n}^{k}$ have length $n$, and $\left|\mathcal{S}_{n}^{k}\right|=\left(\begin{array}{l}n \\ k\end{array}\right)(n-k) !=n ! / k !$. Note that $\mathcal{S}_{n}^{0}$ is the familiar symmetric group $\mathcal{S}_{n}$. For a set $H \subset[n]$ of size $k$, we let $\mathcal{S}_{n}^{H}$ denote the set of partial permutations $\pi_{1} \cdots \pi_{n} \in \mathcal{S}_{n}^{k}$ such that $\pi_{i}=\diamond$ if and only if $i \in H$. We remark that our notion of partial permutations is somewhat reminiscent of the notion of insertion encoding of permutations, introduced by Albert et al. [ALR05]. However, the interpretation of holes in the two settings is different.

In this paper, we extend the classical notion of pattern-avoiding permutations to the more general setting of partial permutations. Let us first recall some definitions related to pattern avoidance on permutations. Let $V=\left\{v_{1}, \ldots, v_{n}\right\}$ with $v_{1}<\cdots<v_{n}$ be any finite subset of $\mathbb{N}$. The standardization of a permutation $\pi$ on $V$ is the permutation st $(\pi)$ on $[n]$ obtained from $\pi$ by replacing the letter $v_{i}$ with the letter $i$. As an example, $\operatorname{st}(19452)=15342$. Given $p \in \mathcal{S}_{k}$ and $\pi \in \mathcal{S}_{n}$, an occurrence of $p$ in $\pi$ is a subword $\omega=\pi_{i(1)} \cdots \pi_{i(k)}$ of $\pi$ such that st $(\omega)=p$; in this context $p$ is called a pattern. If there are no occurrences of $p$ in $\pi$ we also say that $\pi$ avoids $p$. Two patterns $p$ and $q$ are called Wilf-equivalent if for each $n$, the number of $p$-avoiding permutations in $\mathcal{S}_{n}$ is equal to the number of $q$-avoiding permutations in $\mathcal{S}_{n}$.

Let $\pi \in \mathcal{S}_{n}^{k}$ be a partial permutation and let $i(1)<\cdots<i(n-k)$ be the indices of the non-hole elements of $\pi$. A permutation $\sigma \in \mathcal{S}_{n}$ is an extension of $\pi$ if

$$
\operatorname{st}\left(\sigma_{i(1)} \cdots \sigma_{i(n-k)}\right)=\pi_{i(1)} \cdots \pi_{i(n-k)} .
$$

For example, the partial permutation $2 \diamond 1$ has three extensions, namely 312,321 and 231 . In general, the number of extensions of $\pi \in \mathcal{S}_{n}^{k}$ is $\left(\begin{array}{l}n \\ k\end{array}\right) k !=n ! /(n-k) !$.

We are now ready to define pattern avoidance on partial permutations. We say that $\pi \in \mathcal{S}_{n}^{k}$ avoids the pattern $p \in \mathcal{S}_{\ell}$ if each extension of $\pi$ avoids $p$. For example, $\pi=32 \diamond 154$ avoids 1234 , but $\pi$ does not avoid 123: the permutation 325164 is an extension of $\pi$ and it contains two occurrences of 123. Let $\mathcal{S}_{n}^{k}(p)$ be the set of all the partial permutations in $\mathcal{S}_{n}^{k}$ that avoid $p$, and let $s_{n}^{k}(p)=\left|\mathcal{S}_{n}^{k}(p)\right|$. Similarly, if $H \subseteq[n]$ is a set of indices, then $\mathcal{S}_{n}^{H}(p)$ is the set of $p$-avoiding permutations in $\mathcal{S}_{n}^{H}$, and $s_{n}^{H}(p)$ is its cardinality.

We say that two patterns $p$ and $q$ are $k$-Wilf-equivalent if $s_{n}^{k}(p)=s_{n}^{k}(q)$ for all $n$. Notice that 0 -Wilf equivalence coincides with the standard notion of Wilf equivalence. We also say that two patterns $p$ and 
$q$ are $\star$-Wilf-equivalent if $p$ and $q$ are $k$-Wilf-equivalent for all $k \geq 0$. Two patterns $p$ and $q$ are strongly $k$-Wilf-equivalent if $s_{n}^{H}(p)=s_{n}^{H}(q)$ for each $n$ and for each $k$-element subset $H \subseteq[n]$. Finally, $p$ and $q$ are strongly $\star$-Wilf-equivalent if they are strongly $k$-Wilf-equivalent for all $k \geq 0$.

We note that although strong $k$-Wilf equivalence implies $k$-Wilf-equivalence, and strong $\star$-Wilf equivalence implies $\star$-Wilf equivalence, the converse implications are not true. Consider for example the patterns $p=1342$ and $q=2431$. A partial permutation avoids $p$ if and only if its reverse avoids $q$, and thus $p$ and $q$ are $\star$-Wilf-equivalent. However, $p$ and $q$ are not strongly 1-Wilf-equivalent, and hence not strongly $\star$-Wilf equivalent either. To see this, we fix $H=\{2\}$ and easily check that $s_{5}^{H}(p)=13$ while $s_{5}^{H}(q)=14$.

\section{Our Results}

The main goal of this paper is to establish criteria for $k$-Wilf equivalence and $\star$-Wilf equivalence of permutation patterns. We are able to show that most pairs of Wilf-equivalent patterns that were discovered so far are in fact $\star$-Wilf-equivalent. The only exception is the pair of patterns $p=2413$ and $q=1342$. Although these patterns are known to be Wilf-equivalent [Sta94], they are neither 1-Wilf-equivalent nor 2-Wilf equivalent (see Section 6 .

Many of our arguments rely on a close relationship between partial permutations and partial 01-fillings of Ferrers diagrams. These fillings are introduced in Section 2, where we also establish the link between partial fillings and partial permutations.

Our first main result is Theorem 5 in Section 3, which states that a permutation pattern of the form $123 \cdots \ell X$ is strongly $\star$-Wilf-equivalent to the pattern $\ell(\ell-1) \cdots 321 X$, where $X=x_{\ell+1} x_{\ell+2} \cdots x_{m}$ is any permutation of $\{\ell+1, \ldots, m\}$. This theorem is a strengthening of a result of Backelin, West and Xin [BWX07], who show that patterns of this form are Wilf-equivalent. Our proof is based on a different argument than the original proof of Backelin, West and Xin. The main ingredient of our proof is an involution on a set of fillings of Ferrers diagrams, discovered by Krattenthaler [Kra06]. We adapt this involution to partial fillings and use it to obtain a bijective proof of our result.

Our next main result is Theorem 6 in Section 4, which states that for any permutation $X$ of the set $\{4,5, \ldots, k\}$, the two patterns $312 X$ and $231 X$ are strongly $\star$-Wilf-equivalent. This is also a refinement of an earlier result involving Wilf equivalence, due to Stankova and West [SW02]. As in the previous case, the refined version requires a different proof than the weaker version.

In Section 5 we study the $k$-Wilf equivalence of patterns whose length is small in terms of $k$. It is not hard to see that all patterns of length $\ell$ are $k$-Wilf equivalent whenever $\ell \leq k+1$, because $s_{n}^{k}(p)=0$ for every such pattern $p$. Thus, the shortest patterns that exhibit nontrivial behaviour are the patterns of length $k+2$. For these patterns, we show that $k$-Wilf equivalence yields a new characterization of Baxter permutations: a pattern $p$ of length $k+2$ is a Baxter permutation if and only if $s_{n}^{k}(p)=\left(\begin{array}{l}n \\ k\end{array}\right)$. For any non-Baxter permutation $q$ of length $k+2, s_{n}^{k}(q)$ is strictly smaller than $\left(\begin{array}{l}n \\ k\end{array}\right)$ and is in fact a polynomial in $n$ of degree at most $k-1$.

In Section 6, we focus on explicit enumeration of $s_{n}^{k}(p)$ for small patterns $p$. We obtain explicit closedform formulas for $s_{n}^{k}(p)$ for every $p$ of length at most four and every $k \geq 1$.

In view of the space constraints, most of the proofs have been omitted from this extended abstract.

\section{An example: monotone patterns}

Before we present our main results, let us illustrate the concept of pattern-avoiding partial permutations on the example of partial permutations avoiding the monotone pattern $12 \cdots \ell$. Let $\pi \in \mathcal{S}_{n}^{k}$, and let 
$\pi^{\prime} \in \mathcal{S}_{n-k}$ be the permutation obtained from $\pi$ by deleting all $\diamond$ 's. Note that $\pi$ avoids the pattern $12 \cdots \ell$ if and only if $\pi^{\prime}$ avoids $12 \cdots(\ell-k)$. Thus,

$$
s_{n}^{k}(12 \cdots \ell)=\left(\begin{array}{l}
n \\
k
\end{array}\right) s_{n}^{0}(12 \cdots(\ell-k)),
$$

where $\left(\begin{array}{l}n \\ k\end{array}\right)$ counts the possibilities of placing $k \diamond$ 's. For instance, if $\ell=k+3$ then $s_{n}^{k}(12 \cdots \ell)=$ $\left(\begin{array}{l}n \\ k\end{array}\right) s_{n}^{0}(123)$, and it is well known that $s_{n}^{0}(123)=C_{n}$, the $n$-th Catalan number. We remark that for general $\ell$, Regev [Reg81] found an asymptotic formula for $s_{n}^{0}(12 \cdots \ell)$, which can be used to obtain a (rather complicated) asymptotic formula for $s_{n}^{k}(12 \cdots \ell)$ as $n$ tends to infinity.

\section{Partial fillings}

In this section, we introduce the necessary definitions related to partial fillings of Ferrers diagrams. These notions will later be useful in our proofs of $\star$-Wilf equivalence of patterns.

Let $\lambda=\left(\lambda_{1} \geq \lambda_{2} \geq \cdots \geq \lambda_{k}\right)$ be a non-increasing sequence of $k$ nonnegative integers. A Ferrers diagram with shape $\lambda$ is a bottom-justified array $D$ of cells arranged into $k$ columns, such that the $j$-th column from the left has exactly $\lambda_{j}$ cells. Note that our definition of Ferrers diagram is slightly more general than usual, in that we allow columns with no cells. If each column of $D$ has at least one cell, then we call $D$ a proper Ferrers diagram. Every row of a Ferrers diagram $D$ has nonzero length (while we allow columns of zero height). If all the columns of $D$ have zero height-in other words, $D$ has no rows-then $D$ is called degenerate.

For the sake of consistency, we assume throughout this paper that the rows of each diagram and each matrix are numbered from bottom to top, with the bottom row having number 1. Similarly, the columns are numbered from left to right, with column 1 being the leftmost column.

By cell $(i, j)$ of a Ferrers diagram $D$ we mean the cell of $D$ that is the intersection of $i$-th row and $j$-th column of the diagram. We assume that the cell $(i, j)$ is a unit square whose corners are lattice points with coordinates $(i-1, j-1),(i, j-1),(i-1, j)$ and $(i, j)$. The point $(0,0)$ is the bottom-left corner of the whole diagram. We say a diagram $D$ contains a lattice point $(i, j)$ if either $j=0$ and the first column of $D$ has height at least $i$, or $j>0$ and the $j$-th column of $D$ has height at least $i$. A point $(i, j)$ of the diagram $D$ is a boundary point if the cell $(i+1, j+1)$ does not belong to $D$ (see Figure 1 ). Note that a Ferrers diagram with $r$ rows and $c$ columns has $r+c+1$ boundary points.

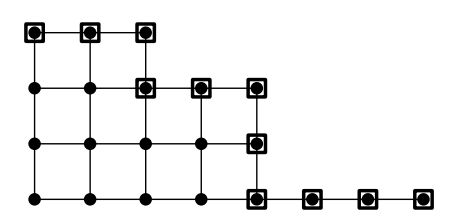

Fig. 1: A Ferrers diagram with shape $(3,3,2,2,0,0,0)$. The black dots represent the points. The black dots in squares are the boundary points.

A 01-filling of a Ferrers diagram assigns to each cell the value 0 or 1. A 01-filling is transversal if each row and each column has exactly one 1-cell. A 01-filling is sparse if each column and each row has at 
most one 1-cell. A permutation $p=p_{1} p_{2} \cdots p_{\ell} \in \mathcal{S}_{\ell}$ can be represented by a permutation matrix which is a 01 -matrix of size $\ell \times \ell$, whose cell $(i, j)$ is equal to 1 if and only if $p_{j}=i$. If there is no risk of confusion, we abuse terminology by identifying a permutation pattern $p$ with the corresponding permutation matrix.

Note that a permutation matrix is a transversal filling of a diagram with square shape.

Let $P$ be a permutation matrix of size $n \times n$, and let $F$ be a sparse filling of a Ferrers diagram. We say that $F$ contains $P$ if $F$ has a (not necessarily contiguous) square subdiagram of size $n \times n$ which induces in $F$ a subfilling equal to $P$. This notion of containment generalizes usual permutation containment.

We now extend the notion of partial permutations to partial fillings of diagrams. Let $D$ be a Ferrers diagram with $k$ columns. Let $H$ be a subset of columns of $D$. Let $\phi$ be a function that assigns to every cell of $D$ one of the three symbols 0,1 and $\diamond$, such that every cell in a column belonging to $H$ is filled with $\diamond$, while every cell in a column not belonging to $H$ is filled with 0 or 1 . The pair $F=(\phi, H)$, will be referred to as a partial 01-filling (or a partial filling) of the diagram D. See Figure 2. The columns from the set $H$ will be called the $\diamond$-columns of $F$, while the remaining columns will be called the standard columns. Observe that if the diagram $D$ has columns of height zero, then $\phi$ itself is not sufficient to determine the filling $F$, because it does not allow us to determine whether the zero-height columns are $\diamond$-columns or standard columns. For our purposes, it is necessary to distinguish between partial fillings that differ only by the status of their zero-height columns.

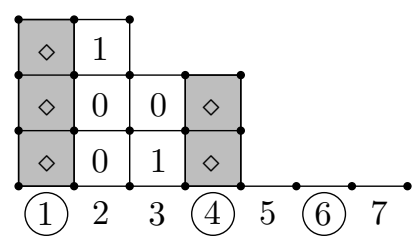

Fig. 2: A partial filling with $\diamond$-columns 1,4 and 6 .

We say that a partial 01-filling is transversal if every row and every standard column has exactly one 1-cell, and we say that a partial 01-filling is sparse if every row and every standard column has at most one 1-cell. A partial 01-matrix is a partial filling of a (possibly degenerate) rectangular diagram .

There is a natural correspondence between partial permutations and transversal partial 01-matrices. Let $\pi \in \mathcal{S}_{n}^{k}$ be a partial permutation. A partial permutation matrix representing $\pi$ is a partial 01-matrix $P$ with $n-k$ rows and $n$ columns, with the following properties:

- If the $j$-th symbol of $\pi$ is $\diamond$, then the $j$-th column of $P$ is a $\diamond$-column.

- If the $j$-th symbol of $\pi$ is a number $i$, then the $j$-th column is a standard column. Also, the cell in column $j$ and row $i$ is filled with 1 , and the remaining cells in column $j$ are filled with 0 's.

To define pattern-avoidance for partial fillings, we first introduce the concept of substitution into a $\diamond-$ column, which is analogous to substituting a number for a $\diamond$ in a partial permutation. The idea is to insert a new row with a 1-cell in the $\diamond$-column; this increases the height of the diagram by one. Let us now describe the substitution formally.

Let $F$ be a partial filling of a Ferrers diagram with $m$ columns. Assume that the $j$-th column of $F$ is a $\diamond$-column. Let $h$ be the height of the $j$-th column. A substitution into the $j$-th column is an operation consisting of the following steps: 
1. Choose a number $i$, with $1 \leq i \leq h+1$.

2. Insert a new row into the diagram, between rows $i-1$ and $i$. The newly inserted row must not be longer than the $(i-1)$-th row, and it must not be shorter than the $i$-th row, so that the new diagram is still a Ferrers diagram. If $i=1$, we also assume that the length of the new row is at most $m$, so that the number of columns does not increase during the substitution.

3. Fill all the cells in column $j$ with the symbol 0 , except for the cell in the newly inserted row, which is filled with 1 . Remove column $j$ from the set of $\diamond$-columns.

4. Fill all the remaining cells of the new row with 0 if they belong to a standard column, and with $\diamond$ if they belong to a $\diamond$-column.

Figure 3 illustrates an example of substitution.
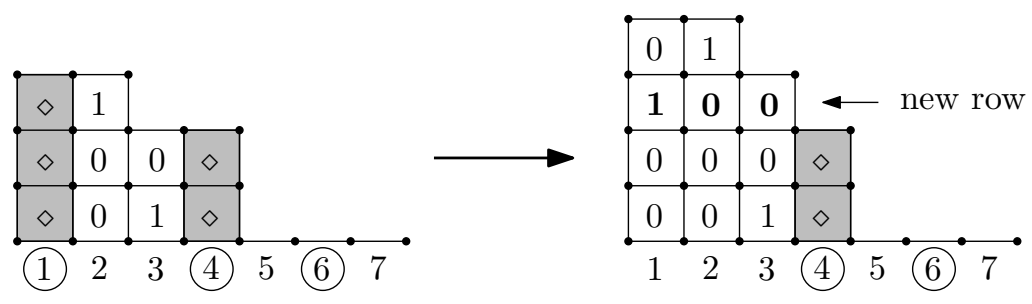

Fig. 3: A substitution into the first column of a partial filling, involving an insertion of a new row between the second and third rows of the original partial filling.

Note that a substitution into a partial filling increases the number of rows by 1 . A substitution into a transversal (resp. sparse) partial filling produces a new transversal (resp. sparse) partial filling. A partial filling $F$ with $k \diamond$-columns can be transformed into a (non-partial) filling $F^{\prime}$ by a sequence of $k$ substitutions; we then say that $F^{\prime}$ is an extension of $F$.

Let $P$ be a permutation matrix. We say that a partial filling $F$ of a Ferrers diagram avoids $P$ if every extension of $F$ avoids $P$. Note that a partial permutation $\pi \in S_{k}^{n}$ avoids a permutation $p$, if and only if the partial permutation matrix representing $\pi$ avoids the permutation matrix representing $p$.

We say that two permutation matrices $P$ and $Q$ are shape- $\star$-Wilf-equivalent, if for every Ferrers diagram $D$ there is a bijection between $P$-avoiding and $Q$-avoiding partial transversal fillings of $D$ that preserves the set of $\diamond$-columns. Observe that if two permutations are shape- $\star$-Wilf-equivalent, then they are also strongly $\star$-Wilf-equivalent, because a partial permutation is a special case of a partial transversal filling of a Ferrers diagram.

The notion of shape- $\star$-Wilf-equivalence is motivated by the following proposition, which extends an analogous result of Babson and West [BW00] for shape-Wilf-equivalence of non-partial permutations.

Proposition 1 Let $P$ and $Q$ be shape- $\star$-Wilf-equivalent permutations, let $X$ be an arbitrary permutation. Then the two permutations $\left(\begin{array}{ll}0 & X \\ P & 0\end{array}\right)$ and $\left(\begin{array}{cc}0 & X \\ Q & 0\end{array}\right)$ are strongly $\star$-Wilf-equivalent.

Due to space constraints, the proof of Proposition 1 is omitted in this extended abstract. 


\section{Strong $\star$-Wilf-equivalence of $12 \cdots \ell X$ and $\ell(\ell-1) \cdots 1 X$}

We will use Proposition 1 as the main tool to prove strong $\star$-Wilf equivalence. To apply the proposition, we need to find pairs of shape- $\star$-Wilf-equivalent patterns. A family of such pairs is provided by the next proposition, which extends previous results of Backelin, West and Xin [BWX07].

Proposition 2 Let $I_{\ell}=12 \cdots \ell$ be the identity permutation of order $\ell$, and let $J_{\ell}=\ell(\ell-1) \cdots 21$ be the anti-identity permutation of order $\ell$. The permutations $I_{\ell}$ and $J_{\ell}$ are shape-丸-Wilf-equivalent.

Before sketching the proof of this proposition, we introduce some notation and terminology. Let $F$ be a partial filling with $r$ rows and $c$ columns. Let $i$ and $j$ be numbers such that the point $(i, j)$ is in $F$. Let $F(\leq i, \leq j)$ denote the submatrix of $F$ whose bottom-left corner is the point $(0,0)$ and whose topright corner is the point $(i, j)$; in other words, $F(\leq i, \leq j)$ is the intersection of the bottom $i$ rows with the leftmost $j$ columns of $F$. We assume that $F(\leq i, \leq 0)$ is the empty matrix, while $F(\leq 0, \leq j)$ is the degenerate matrix with no rows but with $j$ columns of zero height.

Let $F$ be a sparse partial filling of a Ferrers diagram, and let $(i, j)$ be a boundary point of $F$. Let $h(F, j)$ denote the number of $\diamond$-columns among the first $j$ columns of $F$. Let $I(F, i, j)$ denote the largest integer $\ell$ such that the partial matrix $F(\leq i, \leq j)$ contains $I_{\ell}$. Similarly, let $J(F, i, j)$ denote the largest $\ell$ such that $F(\leq i, \leq j)$ contains $J_{\ell}$.

We let $F_{0}$ denote the (non-partial) sparse filling obtained by replacing all the symbols $\diamond$ in $F$ by zeros. Let us state without proof the following simple observation.

Observation 3 Let $F$ be a sparse partial filling.

1. F contains a permutation matrix $P$ if and only if $F$ has a boundary point $(i, j)$ such that $F(\leq i, \leq$ j) contains $P$.

2. For any boundary point $(i, j)$, we have $I(F, i, j)=h(F, j)+I\left(F_{0}, i, j\right)$ and $J(F, i, j)=h(F, j)+$ $J\left(F_{0}, i, j\right)$.

The key to the proof of Proposition 2 is the following theorem, which follows directly from the powerful results of Krattenthaler [Kra06] obtained using the theory of growth diagrams.

Theorem 4 (Krattenthaler [Kra06]) Let $D$ be a Ferrers diagram. There is a bijective mapping $\kappa$ from the set of all (non-partial) sparse fillings of D onto itself, with the following properties.

1. For any boundary point $(i, j)$ of $D$, and for any sparse filling $F$, we have $I(F, i, j)=J(\kappa(F), i, j)$ and $J(F, i, j)=I(\kappa(F), i, j)$.

2. The mapping $\kappa$ preserves the number of 1-cells in each row and column. In other words, if a row (or column) of a sparse filling $F$ has no 1-cell, then the same row (or column) of $\kappa(F)$ has no 1 -cell either.

In Krattenthaler's paper, the results are stated in terms of proper Ferrers diagrams. However, the bijection obviously extends to Ferrers diagrams with zero-height columns as well. This is because adding zero-height columns to a (non-partial) filling does not affect pattern containment.

From the previous theorem, we easily obtain the proof of the main proposition in this section.

Proof of Proposition 2 Let $D$ be a Ferrers diagram. Let $F$ be an $I_{\ell}$-avoiding transversal partial filling of $D$. Let $F_{0}$ be the sparse filling obtained by replacing all the $\diamond$ characters of $F$ by zeros. Define 
$G_{0}=\kappa\left(F_{0}\right)$, where $\kappa$ is the bijection from Theorem 4 . Note that all the $\diamond$-columns of $F$ are filled with zeros both in $F_{0}$ and $G_{0}$. Let $G$ be the sparse partial filling obtained from $G_{0}$ by replacing zeros with $\diamond$ in all such columns. Then $G$ is a sparse partial filling with the same set of $\diamond$-columns as $F$.

We see that for any boundary point $(i, j)$ of the diagram $D, h(F, j)=h(G, j)$. By the properties of $\kappa$, we further obtain $I\left(F_{0}, i, j\right)=J\left(G_{0}, i, j\right)$. In view of Observation 3 this implies that $G$ is a $J_{\ell^{-}}$ avoiding filling. It is clear that this construction can be inverted, thus giving the required bijection between $I_{\ell}$-avoiding and $J_{\ell}$-avoiding transversal partial fillings of $D$.

Combining Proposition 1 with Proposition 2, we get directly the main result of this section.

Theorem 5 For any $\ell \leq m$, and for any permutation $X$ of $\{\ell+1, \ldots, m\}$, the permutation pattern $123 \cdots(\ell-1) \ell X$ is strongly $\star$-Wilf-equivalent to the pattern $\ell(\ell-1) \cdots 21 X$.

Notice that Theorem 5 implies, among other things, that all the patterns of size three are strongly $\star$-Wilf-equivalent.

\section{Strong $\star$-Wilf-equivalence of $312 X$ and $231 X$}

We will now focus on the two patterns 312 and 231. The main result of this section is the following theorem.

Theorem 6 The patterns 312 and 231 are shape- $\star$-Wilf-equivalent. By Proposition 1 this implies that for any permutation $X$ of the set $\{4,5, \ldots, m\}$, the two permutations $312 X$ and $231 X$ are strongly $\star-$ Wilf-equivalent.

Theorem 6generalizes a result of Stankova and West [SW02], who have shown that 312 and 231 are shape-Wilf equivalent. The original proof of Stankova and West [SW02] is rather complicated, and does not seem to admit a straightforward generalization to the setting of shape- $\star$-Wilf-equivalence. Our proof of Theorem 6 is different from the argument of Stankova and West, and it is based on a bijection of Jelínek [Jel07], obtained in the context of pattern-avoiding ordered matchings.

Due to space limitations, we omit the whole very long proof from this extended abstract.

\section{The $k$-Wilf-equivalence of patterns of length $k+2$}

We will now consider the structure of pattern-avoiding partial permutations in which the number of $\diamond$ 's is close to the length of the forbidden pattern.

Let us begin by an easy observation.

Observation 7 Assume that $p$ is a pattern of length $\ell$. Any partial permutation with at least $\ell$ occurrences of $\diamond$ contains $p$. Almost as obviously, a partial permutation with $\ell-1$ occurrences of $\diamond$ and of length at least $\ell$, contains $p$ as well. In particular, for every $k \geq \ell-1$, we have $s_{n}^{k}(p)=0$, and hence all the patterns of length $\ell$ are $k$-Wilf-equivalent.

In the rest of this section, we will deal with $k$-Wilf-equivalence of patterns of length $\ell=k+2$.

As we will see, an important part in $k$-Wilf-equivalence is played by Baxter permutations, which are defined as follows.

Definition 8 A permutation $p \in \mathcal{S}_{\ell}$ is called a Baxter permutation, if there is no four-tuple of indices $a<b<c<d \in[\ell]$ such that 
- $c=b+1$, and

- the subpermutation $p_{a}, p_{b}, p_{c}, p_{d}$ is order-isomorphic to 2413 or to 3142.

In the terminology of Babson and Steingrimsson [BSO0], Baxter permutations are exactly the permutations avoiding simultaneously the two patterns 2-41-3 and 3-14-2.

Baxter permutations have been introduced by G. Baxter [Bax64] in 1964. They were originally encountered in the context of common fixed points of commuting continuous functions [Bax64, Boy81]. Later, it has been discovered that Baxter permutations are also closely related to other combinatorial structures, such as plane bipolar orientations [BBMF08], noncrossing triples of lattice paths [FFNO08], and standard Young tableaux [DG96]. An explicit formula for the number of Baxter permutations has been found by Chung et al. [CGJK78], with several later refinements [Mal79, Vie81, DG98].

It is not hard to see that for any pattern of length $\ell=k+2$, and for any $n$ from the set $\{k, k+1, k+2\}$, we always have $s_{n}^{k}(p)=\left(\begin{array}{l}n \\ k\end{array}\right)$. Thus, for these small values of $n$, all patterns have the same behavior. However, for all larger values of $n$, the Baxter patterns are separated from the rest, as the next proposition and theorem show. We omit the proofs of these results.

Proposition 9 Let $p$ be a permutation pattern of size $\ell$, and let $k=\ell-2$. The following statements are equivalent.

\section{The pattern $p$ is a Baxter permutation.}

2. For each $n \geq k$ and each $k$-element subset $H \subseteq[n], s_{n}^{H}(p)=1$.

3. For $n=k+3$ and each $k$-element subset $H \subseteq[n], s_{n}^{H}(p)=1$.

4. There exists $n \geq k+3$ such that for each $k$-element subset $H \subseteq[n], s_{n}^{H}(p)=1$.

Theorem 10 Let $p \in \mathcal{S}_{\ell}$ be a permutation pattern. Let $k=\ell-2$. If $p$ is a Baxter permutation then $s_{n}^{k}(p)=\left(\begin{array}{l}n \\ k\end{array}\right)$ for each $n \geq k$. If $p$ is not a Baxter permutation, then $s_{n}^{k}(p)<\left(\begin{array}{l}n \\ k\end{array}\right)$ whenever $n \geq k+3$. Moreover, all the Baxter permutations are strongly $k$-Wilf equivalent.

We remark that by a slightly more careful analysis of the arguments leading to Proposition 9 and Theorem 10, we could give a stronger upper bound for $s_{n}^{k}(p)$ when $p$ is not a Baxter permutation. In particular, it is not hard to show that in that case, $s_{n}^{k}(p)$ is eventually equal to a polynomial in $n$ of degree at most $k-1$, with coefficients depending on $k$.

\section{Short patterns}

In the rest of this paper, we focus on explicit formulas for $s_{n}^{k}(p)$, where $p$ is a pattern of length $\ell$. We may assume that $k<\ell-1$, and $\ell>2$, since for any other values of $(k, \ell)$ the enumeration is trivial (see Observation 7). We also restrict ourselves to $k \geq 1$, since the case $k=0$, which corresponds to classical pattern-avoidance in permutations, has already been extensively studied [Bó4].

For a pattern $p$ of length three, the situation is very simple. Theorem 10 implies that $s_{n}^{1}(p)=n$, since all permutations of length three are Baxter permutations.

Let us now deal with patterns of length four. In Figure 4 , we depict the $k$-Wilf equivalence classes, where the four rows, top to bottom, correspond to the four values $k=0,1,2,3$. Since all the $k$-Wilf 
equivalences are closed under complements and reversals (but not inversions), we represent the 24 patterns of length four by eight representatives, one from each symmetry class. For instance, $\{1342,1423\}$ in the second row represents the union of $\{1342,2431,3124,4213\}$ and $\{1423,2314,3241,4132\}$.

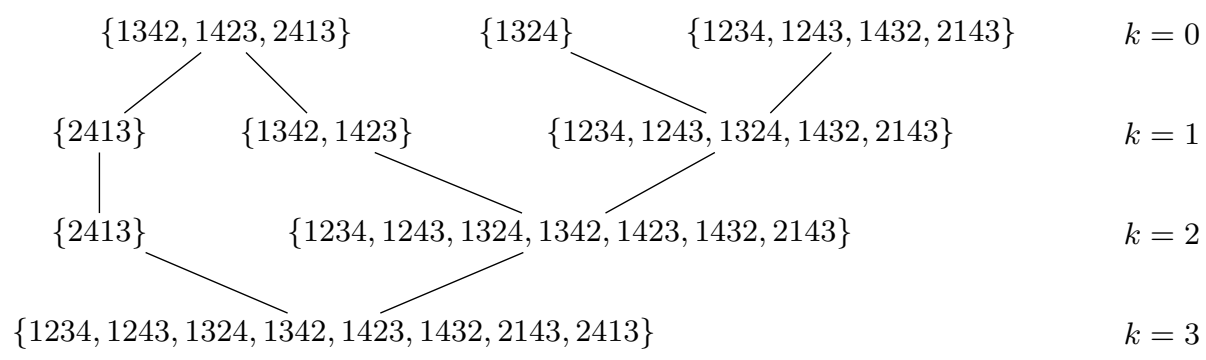

Fig. 4: The $k$-Wilf-equivalence classes of permutations of size 4 .

All patterns $p$ of length four except 2413 and 3142 are Baxter permutations, and hence they satisfy $s_{n}^{2}(p)=\left(\begin{array}{c}n \\ 2\end{array}\right)$ by Theorem 10 It is possible to show that $s_{n}^{2}(2413)=s_{n}^{2}(3142)=3 n-6$. We omit the details of this routine argument in this extended abstract.

In the rest of the paper, we deal with 1-Wilf equivalence of patterns of length four, and with the enumeration of the corresponding avoidance classes. Theorem 5 and symmetry arguments imply that all the patterns 1234, 1243, 1432 and 2143 are strongly $\star$-Wilf-equivalent, and Theorem 6 with appropriate symmetry arguments shows that 1342 and 1423 are strongly $\star$-Wilf-equivalent as well. The only case not covered by these general theorems is the 1-Wilf equivalence of 1324 and 1234, which is handled separately by the next proposition.

Proposition 11 The patterns 1234 and 1324 are strongly 1-Wilf-equivalent.

The proof of Proposition 11 is omitted.

Let us now state the formulas for $s_{n}^{1}(p)$, where $p$ has length four. The proofs are omitted.

Theorem 12 The number of partial permutations of length $n \geq 1$ with a single hole, avoiding a pattern of length four, is given by these formulas:

- $s_{n}^{1}(1234)=s_{n}^{1}(1243)=s_{n}^{1}(1324)=s_{n}^{1}(1432)=s_{n}^{1}(2143)=\left(\begin{array}{c}2 n-2 \\ n-1\end{array}\right)$,

- $s_{n}^{1}(1342)=s_{n}^{1}(1423)=\left(\begin{array}{c}2 n-2 \\ n-1\end{array}\right)-\left(\begin{array}{c}2 n-2 \\ n-5\end{array}\right)$, and

- $s_{n}^{1}(2413)=\frac{2}{n+1}\left(\begin{array}{c}2 n \\ n\end{array}\right)-2^{n-1}$.

\section{Directions of further research}

We have shown that classical Wilf equivalence may be regarded as a special case in a hierarchy of $k$-Wilf equivalence relations, and that many properties previously established in the context of Wilf equivalence can be generalized to all the $k$-Wilf equivalences. In many situations, understanding the $k$-Wilf equivalence class of a given pattern $p$ becomes easier as $k$ increases. In particular, the $k$-Wilf equivalence class of the permutation $p=12 \cdots(k+1)(k+2)$ contains exactly the Baxter permutations of length $k+2$. 
What can we say about the $k$-Wilf equivalence class of the permutation $12 \cdots(k+3)$ ? For $k=0$ and $k=1$ this class contains exactly the layered permutations of length $k+3$. Computer enumeration suggests that the same is true for larger values of $k$ as well, but we have no proof.

\section{References}

[ALR05] Michael H. Albert, Steve Linton, and Nik Ruškuc. The insertion encoding of permutations. Electron. J. Combin, 12(1), 2005. Research paper 47, 31 pp.

[B0́4] M. Bóna. Combinatorics of Permutations. Discrete Mathematics and its Applications. Chapman and Hall/CRC Press, 2004.

[Bax64] G. Baxter. On fixed points of the composite of commuting functions. Proceedings of the American Mathematical Society, 15(6):851 - 855, 1964.

[BB99] J. Berstel and L. Boasson. Partial words and a theorem of Fine and Wilf. Theoretical Computer Science, 218(1):135 - 141, 1999.

[BBMF08] N. Bonichon, M. Bousquet-Mélou, and É. Fusy. Baxter permutations and plane bipolar orientations. Electronic Notes in Discrete Mathematics, 31:69 - 74, 2008. The International Conference on Topological and Geometric Graph Theory.

[BBSGR09] B. Blakeley, F. Blanchet-Sadri, J. Gunter, and N. Rampersad. Developments in Language Theory, volume 5583 of $L N C S$, chapter On the Complexity of Deciding Avoidability of Sets of Partial Words, pages 113 - 124. Springer, 2009.

[Boy81] W. M. Boyce. Baxter permutations and functional composition. Houston Journal of Mathematics, 7(2):175 - 189, 1981.

[BS00] E. Babson and E. Steingrímsson. Generalized permutation patterns and a classification of the Mahonian statistics. Séminaire Lotharingien de Combinatoire, 44:18, 2000.

[BS08] F. Blanchet-Sadri. Algorithmic combinatorics on partial words. Discrete Mathematics and its Applications. Chapman \& Hall/CRC, Boca Raton, FL, 2008.

$\left[\mathrm{BSBK}^{+}\right.$09] F. Blanchet-Sadri, N. C. Brownstein, A. Kalcic, J. Palumbo, and T. Weyand. Unavoidable sets of partial words. Theory of Computing Systems, 45(2):381-406, 2009.

[BW00] E. Babson and J. West. The permutations $123 p_{4} \cdots p_{m}$ and $321 p_{4} \cdots p_{m}$ are Wilfequivalent. Graphs and Combinatorics, 16(4):373 - 380, 2000.

[BWX07] J. Backelin, J. West, and G. Xin. Wilf-equivalence for singleton classes. Advances in Applied Mathematics, 38(2):133 - 148, 2007.

[CGJK78] F. R. K. Chung, R. L. Graham, V. E. Hoggatt Jr., and M. Kleiman. The number of Baxter permutations. J. Combin. Theory Ser. A, 24(3):382 - 394, 1978.

[DG96] S. Dulucq and O. Guibert. Stack words, standard tableaux and Baxter permutations. Discrete Mathematics, 157(1-3):91 - 106, 1996. 
[DG98] S. Dulucq and O. Guibert. Baxter permutations. Discrete Mathematics, 180(1-3):143 - 156, 1998. Proceedings of the 7th Conference on Formal Power Series and Algebraic Combinatorics.

[FFNO08] S. Felsner, E. Fusy, M. Noy, and D. Orden. Bijections for Baxter families and related objects. arXiv:0803.1546, 2008.

[HHK08] V. Halava, T. Harju, and T. Kärki. Square-free partial words. Information Processing Letters, 108(5):290 - 292, 2008.

[HHKS09] V. Halava, T. Harju, T. Kärki, and P. Séébold. Overlap-freeness in infinite partial words. Theoretical Computer Science, 410(8-10):943 - 948, 2009.

[Jel07] V. Jelínek. Dyck paths and pattern-avoiding matchings. European Journal of Combinatorics, 28(1):202 - 213, 2007.

[Kra06] C. Krattenthaler. Growth diagrams, and increasing and decreasing chains in fillings of Ferrers shapes. Advances in Applied Mathematics, 37(3):404 - 431, 2006.

[Leu05] P. Leupold. Partial words for dna coding. In DNA 10, Tenth International Meeting on DNA Computing, volume 3384 of LNCS, pages 224 - 234. Springer-Verlag, Berlin, 2005.

[Mal79] C. L. Mallows. Baxter permutations rise again. Journal of Combinatorial Theory, Series A, 27(3):394-396, 1979.

[Reg81] Amitai Regev. Asymptotic values for degrees associated with strips of Young diagrams. Advances in Mathematics, 41(2):115 - 136, 1981.

[SK01] A. M. Shur and Y. V. Konovalova. On the periods of partial words. In MFCS '01: Proceedings of the 26th International Symposium on Mathematical Foundations of Computer Science, volume 2136 of LNCS, pages 657 - 665. Springer-Verlag, 2001.

[Sta94] Z. Stankova. Forbidden subsequences. Discrete Mathematics, 132(1-3):291-316, 1994.

[SW02] Z. Stankova and J. West. A new class of Wilf-equivalent permutations. J. Algebraic Comb., 15(3):271-290, 2002.

[Vie81] G. Viennot. A bijective proof for the number of Baxter permutations. Séminaire Lotharingien de Combinatoire, 1981. 